\title{
Sequence plus structure plus experimental inquiry: combining the clues to elucidate bacterial kinase function
}

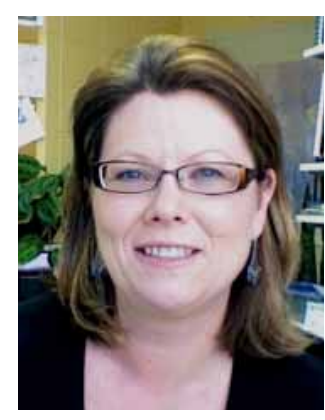

Nangy Martin

$D$ epartment of M icrobiology \& Immunology,

Gastrointestinal D iseases \& Protein Function D iscovery Research G roups, Q ueen's University, Kingston, Ontario, K 7L 3N 6, Canada Tel.: +1 613533 2460; Fax: +1 613533 6796; nancy.martin@queensu.ca

\author{
'As the a mount of genome, \\ transcriptome and proteome \\ data increases... it is important \\ that the scientific community \\ provides experimental \\ evidence to comoborate and \\ ensure the accuracy of these \\ important resources'.
}

The overwhelming amount of data available on the world wide web about many aspects of bacteria gives the impression that we know a great deal about particular bacteria. We do, but what do we know as fact versus conjecture? As of April 2007, there were 1129 Bacterial and 68 Archaeal genome projects listed on the N CBI Entrez website. Combine this wealth of genome information with the array of databases available to analyze both the genomic information and the translated products of these genomes, along with protein structure and protein motif databases, and the impression is that one need not ever approach the bench in order to determine the function of a protein of interest. U nfortunately (or fortunately for those who enjoy working at the bench rather than at a computer), unless the 'founding' information in a particular database is particularily sound and based on experimental evidence, the rapid expansion and automated annotation of genomic sequences can lead to the propagation of masses of information that are not necessarily accurate. Although we increasingly operate in an environment focused on 'omic' (e.g., genomic, proteomic and transcriptomic) approaches, relying solely on sequence or even structural-based similarities does not necessarily lead to understanding protein function. Experimental approaches that examine phenotypic, biochemical and structural properties of the specific genes, transcripts and proteins continue to provide essential information that serves to fundamentally refine the 'omic' data. These concepts can be illustrated using an example of a protein that my laboratory works with, YinE/R doA.
Why study YihE/RdoA?

Part of the impetus for our work is to search for uniquely bacterial systems that can be targeted for antimicrobial therapy which will cause decreased virulence, and YihE/RdoA is a potential candidate. The gene encoding this protein was first annotated as yihE as a result of there being no known function for it at the time of the Escherichia coli physical map construction EcoM ap7 [1]. The yihE gene is part of a two-gene operon consisting of yihE-dsbA, the latter gene coding for a well-studied disulfide oxidoreductase in E. coli $[2,3]$ and Salmonella enterica serovar Typhimurium (S. typhimurium) [4]. We became interested in the function of the yihE gene product as it and D sbA are members of the C px reguIon. C px is a two-component signal transduction pathway that is activated via a variety of stresses that generally affect processes which take place in the bacterial cell envelope, including functions important for maintenance of virulence [5-7]. W hile experimental data demonstrate how $D$ sbA contributed to the bacterial cell's abilities to deal with stress or external environmental conditions, by helping to fold or refold misfolded periplasmically located proteins [8], the role of YinE was unknown. O ur initial investigation of the YinE homolog in S. typhimurium suggested that it actually affects D sbA levels in the cell and we began using the moniker RdoA (regulator of disulfide oxidoreductase A), although we had little idea of the RdoA mechanism at that time [9]. YinE and RdoA share 303/328 similar amino acids, with 282 of these being identical.

\section{Clues to YinE/RdoA function based on genomic databases}

$O$ ne hint of $Y$ ihE/RdoA function came from its annotation as a putative type II homoserine kinase. The origin of this annotation appears to be linked to the extremely limited homology between $Y i h E / R d o A$ and true homoserine kinases (EC 2.7.1.39) found in E. coli and other bacteria, which are part of the threonine biosynthetic pathway that is generally conserved in all bacteria (as described in [10]). In a protein-protein basic local alignment search tool (BLAST) analysis [11] using RdoA as the comparator 
against the Concise BLAST database for microbial genomes, significant matches to 127 proteins that resolve into 85 genus-level clusters are generated. These are variously annotated as hypothetical proteins, predicted kinase, phosphotransferase enzyme, serine protease and aminoglycoside phosphotransferase, and are in genera of the Proteobacteria, along with one genus of the Spirochaetes. YinE/RdoA are also part of COG2334 'putative homoserine kinase type II (protein kinase fold)' along with 31 additional proteins that include representatives from the Euryarchaeota, Cyanobacteria, D einococcus-T hermus, Fusobacteria and Firmicutes, in addition to the majority in the Gramnegative Proteobacteria. Prior to our work, there does not appear to be any experimental evidence supporting any of these annotations.

Does knowing this annotation information help to determine the function of YihE/R doA? For us, the consistent suggestion that these proteins had some sort of kinaselike function prompted us to crystallize and begin to biochemically characterize YinE and RdoA by testing in vitro for kinase activity [12]. While the structure of YinE showed many of the commonly conserved motifs of a protein kinase, to our surprise, the structure is most similar to a eukaryotic choline kinase (PD B IN W 1) from Caenorhabditis elegans or aminoglycoside 3 '-phosphotransferase (APH [3']-IIIa, PDB 1J7I) from Enterococcus faecalis. These two proteins never appear in amino acid sequence homology searches to YinE because there is relatively little amino acid conservation except in regions predicted to interact with ATP and those involved in phosphotransfer. These two proteins also contain 65 fewer amino acid residues than YihE and the majority of its sequence-based homologs. Interestingly, if $\mathrm{APH}\left(3^{\prime}\right)-\mathrm{Ill}$ a is used to conduct protein-protein BLAST analyses, none of the aminoglycoside phosphotransferases previously identified as similar to YihE are retrieved. Choline kinase and APH ( $\left.3^{\prime}\right)$-III a are both small molecule kinases rather than protein kinases. The structures of choline kinase and APH (3')IIla have been extensively analyzed by Scheeff and Bourne [13], who place them in a group of atypical kinases within a phylogenetic tree branch of a protein kinase-like superfamily noting that, of the currently included six candidates, choline kinase and APH ( $\left.3^{\prime}\right)$-III a are most closely related to protein kinases. We propose that YihE, although similar in structure to these small molecule kinases, is in fact a Ser/Thr protein kinase that may represent an evolutionary step between more traditional protein kinase structures and small molecule kinases such as APH (3')-IIIa.

\section{Experimental evidence for Ser/Thr} kinase activity

What is the evidence for YihE/RdoA being Ser/Thr protein kinases? We have demonstrated that both YinE and RdoA are autophosphorylated on serine and threonine residues and are also capable of phosphorylating the non-native substrate myelin basic protein [12]. Recognizing that APH $\left(3^{\prime}\right)$-IIIa has also been shown to act as a Ser/T hr protein kinase in vitro, albeit with extremely low efficiency [14], thorough kinetic analyses will need to be carried out on YinE/RdoA. Our current data show YihE to have a relatively high activity in phosphorylating myelin basic protein, but the native substrate of YihE/RdoA is not currently known and so these studies await the identification of this important molecule(s).

\footnotetext{
'This vast a rray of a ltera tions in gene expression and subsequent phenotypes a ttributable to YihE/RdoA strongly suggests that they play a role in the regulation of seemingly different processes'.
}

Neither deleting nor over-expressing YihE/RdoA alters sensitivity to aminoglycosides such as kanamycin, suggesting that aminoglycoside modification is not the function of YihE/RdoA. The arrangement of the YihE crystal packing provided an interesting clue to potential substrates. The six-residue $\mathrm{C}$-terminal tail of YinE was found to be inserted into the predicted substrate-binding region of the neighboring molecule, forming a natural protein-peptide complex. This shows that a peptide can easily dock within the substrate binding region and further screening for peptide-based analogues of this region is underway.

What do the phenotypes related to YihE/RdoA tell us? We know that RdoA is much more abundant in stationary phase cells, is expressed without induction of the $\mathrm{C} p \mathrm{p}$ pathway and is important in long-term cell survival [12]. We also know that rdoA null strains of $S$. typhimurium are altered in their ability to undergo phase variation of flagellin expression [Richards $M$, M artin N , Unpublished D ata] as well as being derepressed for curli expression [12]. Earlier microarray studies of a yihE null Shigella flexneri showed a wide 
range of genes to be up- or downregulated [15], and follow-up studies determined that LPS synthesis is affected via a downregulation of the galETK operon [16]. These authors also noted a novel cytotoxic activity in the yihE null mutant, suggesting that YinE normally represses expression of this factor. This vast array of alterations in gene expression and subsequent phenotypes attributable to $\mathrm{YinE} / \mathrm{R}$ doA strongly suggests that they play a role in the regulation of seemingly different processes. The simplest explanation fitting these data would be that YihE/RdoA act to phosphorylate and thereby activate or repress an important global regulatory protein.

Looking again at the amino acid sequence comparisons, 28 of the 85 genera that have significant similarity to YinE/RdoA have also retained the $d S D A$ gene immediately downstream of the kinase. These include all of the Order Enterobacteriales homologs (i.e., Shigella, Yers inia, Erwinia, Escherichia and Salmonella) as well as those of additional Gammaproteobacteria of various O rders, some of the more exotic members being the deep-sea eubacterium Photobacte rium profundum, the marine psychrophilic bacterium Colwellia psychrerythraea, and Psychromonas ingrahamii, which was isolated from sea ice and can grow at $-12^{\circ} \mathrm{C}$ [17].

'In comparison with the large
number of euka ryotic kina ses...
the current knowledge of
bacterial kina ses, especially
Ser/Thr kinases, is extremely limited'.

O ne would predict that, whatever the target of YihE/RdoA activity, it is likely to be similar in these organisms due to the high level of sequence, and therefore functional, conservation. These same bacteria also appear to have retained the C $\mathrm{px}$ system, as indicated by the presence of a gene annotated as cpxA, but these data have not been rigorously checked. The remaining 57 YihE/RdoA homologues identified are all between 321 and 359 amino acids in length and do not have consistent neighbors either up- or downstream in their respective genomes. Included in this group are various genera from the Betaproteobacteria, Pseudomonads from the $\mathrm{G}$ ammaproteobacteria, and a few examples from the Deltaproteobacteria such as Bdellovibrio bacteriovorous. Whether or not these more distantly related homologs retain similar activity remains to be tested, although since the proposed catalytic domains for kinase activity are present, this group of proteins are likely to have different substrate specificities, but retain kinase activity.

\section{Conclusion}

There are a large group of proteins in the public domain genomic databases currently annotated as aminoglycoside phosphotransferases that probably are not. After all, why would a deep-sea organism need to modify aminoglycoside antibiotics? Also, YihE/R doA and their homologs do not appear to be homoserine kinases, although they are often annotated as such. It would be most helpful in ongoing annotations efforts if it is clearly indicated on what basis a particular annotation has been based, although with the advent of automated annotation that is not actually rigorously checked, labeling of genes/proteins without direct experimental evidence is bound to continue. As our experimental tools become more and more refined, it is becomingly increasingly obvious that while overview-type data provide clues to understanding biological function, this type of analysis can miss fundamental differences. The 'average result' after all does not apply precisely to any one example - if it did, it would not be an average.

\section{Future perspective}

In comparison with the large number of eukaryotic kinases that have been characterized, the current knowledge of bacterial kinases, especially Ser/T hr kinases, is extremely limited. $D$ ata are beginning to accumulate on different types of bacterial kinases and will undoubtedly show that bacteria also use phosphorylation in signaling networks that function in relaying environmental signals that bacteria must adapt to in order to survive. This is especially important in understanding how virulence determinants are regulated and expressed in different host/infection situations and in developing targeted antimicrobial therapies. As the amount of genome, transcriptome and proteome data increases ever more rapidly, it is important that the scientific community provides experimental evidence to corroborate and ensure the accuracy of these important resources by continuing to carry out essential experiments to validate predictions of function.

\section{Acknowledgement}

The author would like to acknowledge the support of the $\mathrm{N}$ atural Sciences and Engineering Research Council of Canada for providing operating funding. 


\section{Executive summary}

\section{Why study YinE/RdoA?}

- YihE/RdoA and numerous bacterial homologs have not been previously characterized and are annotated with several different potential functions in genomic databases.

- Bacterial kinases are potentially interesting targets for antimicrobial therapy development.

\section{Experimental evidence for Ser/Thr kinase activity}

- YinE/RdoA are bacterial Ser/Thr protein kinases with a novel structure that may represent an evolutionary stage between eukaryotic protein kinases and prokaryotic protein kinase-like small molecule kinases.

\section{Conclusion}

- It is important to understand the amount of conjecture that is used to annotate genomic databases and to realize that amino acid sequence homology does not always predict function.

\section{Future perspective}

- The group of YinE/RdoA-like bacterial kinases are highly conserved in a large group of clinically relevant Gram-negative bacteria. Elucidation of the functions of these types of enzymes will help to determine how bacteria respond to changes in their external environment, such as at an infection site, and thereby provide a better understanding of the appropriate targets for antimicrobial therapy.

\section{Bibliography}

1. Berlyn M K, Low B, Rudd KE: Linkage map of Escherichia coli K-12. In: Escherichia coli and Salmonella (9th Edition). N eidhardt FC (Ed.). ASM Press, Washington, DC, USA 1715-1902 (1996).

2. M essens J, C ollet JF: Pathways of disulfide bond formation in Escherichia coli. Int. J. Biochem. Cell Biol. 38, 1050-1062 (2006).

3. Kadokura H, Katzen F, Beckwith J: Protein disulfide bond formation in prokaryotes. Annu. Rev. Biochem. 72, 111-135 (2003).

4. Turcot I, Ponnampalam TV, Bouwman CW, $M \operatorname{artin} \mathrm{NL}$ : I solation and characterization of a chromosomally encoded disulphide oxidoreductase from Salmonella enterica serovar Typhimurium. Can. J. M icrobiol. 47, 711-721 (2001).

5. Dorel $C$, Lejeune $P$, Rodrigue A: The Cpx system of Escherichia coli, a strategic signaling pathway for confronting adverse conditions and for settling biofilm communities? Res. M icrobiol. 157, 306-314 (2006).

6. Nevesinjac AZ, Raivio TL: The C px envelope stress response affects expression of the type IV bundle-forming pili of enteropathogenic Escherichia coli. J. Bacteriol. 187, 672-686 (2005).
7. Ruiz N , Silhavy T J : Sensing external stress: watchdogs of the Escherichia coli cell envelope. Curr. O p. M icrob. 8, 122-126 (2005).

8. Raivio TL: Envelope stress responses and $\mathrm{Gram}$-negative bacterial pathogenesis. M olec. M icrobiol. 56, 1119-1128 (2005).

9. Suntharalingam $P$, Spencer $H, G$ allant $C$, $M$ artin N L: Salmonella typhimurium rdoA is growth phase regulated and involved in relaying $\mathrm{Cpx}$ induced signals. J. Bacteriol. 185, 432-443 (2003).

10. Bareich D, Koteva K, N azi I, Wright GD: Small molecule functional discrimination of the kinases required for the microbial synthesis of threonine and isoleucine. Bioorg. M ed. Chem. 12, 807-815 (2004).

11. Altschul SF, Gish W, M iller W, M yers EW, Lipman DJ: Basic local alignment search tool. J. M ol. Biol. 215, 403-410 (1990).

12. Zheng J, HeC, Singh VK, M artin N L, Jia Z: C rystal structure of a novel prokaryotic Ser/T hr kinase and its implication in the $C p x$ stress response pathway. M olec. M icrobiol. 63, 1360-1371 (2007)
13. Scheeff ED, Bourne PE: Structural evolution of the protein kinase-like superfamily. PLOS Comput. Biol. 1, E49 (2005).

14. D aigle D M, M CK ay GA, Thompson PR, Wright GD : Aminoglycoside antibiotic phosphotransferases are al so serine protein kinases. Chem. Biol. 6, 11-18 (1999).

15. Li M S, Kroll JS, Yu J: Influence of the yihE gene of Shigella flexneri on global gene expression: on analysis using D N A arrays. Biochem. Biophys. Res. Commun. 288, 91-100 (2001).

16. Edwards-J ones B, Langford PR, K roll JS, Yu J: The role of the Shigella flexneri yihE gene in LPS synthesis and virulence. M icrobiology 150, 1079-1084 (2004).

17. Breezee J, Cady N, Staley JT: Subfreezing growth of the sea ice bacterium "Psychromonasingrahamii". M icrob. Ecol. 47, 300-304 (2004).

Affiliation

- Nancy L M artin

D epartment of M icrobiology \& Immunology, Gastrointestinal D iseases\& Protein Function D iscovery Research Groups, Q ueen's U niversity, Kingston, Ontario, K $7 \mathrm{~L}$ 3N 6, Canada Tel.: +1 613533 2460;

Fax: +1 613533 6796;

nancy.martin@queensu.ca 